\title{
On the Use of Multiple Probe Insertions at the Same Site for Repeated Intracerebral Microdialysis Experiments in the Nigrostriatal Dopamine System of Rats
}

\author{
*Dianne M. Camp and ${ }^{*} \uparrow$ Terry E. Robinson \\ *Department of Psychology and $\uparrow$ Neuroscience Program, The University of Michigan, Ann Arbor, Michigan, U.S.A.
}

\begin{abstract}
The effects of implantation of a dialysis probe into the striatum of awake rats on indices of dopamine (DA) and serotonin neurotransmission were assessed, first over $24 \mathrm{~h}$ following initial insertion of a probe, and then again following reinsertion of a probe at the same site 1 week later. It was found that the basal concentration of DA in dialysate stabilized within $20-40$ min after probe implantation, although DA showed a modest decline $24 \mathrm{~h}$ later. There was, however, no significant difference in basal DA between two test sessions separated by 1 week. On the other hand, the basal concentrations of the DA metabolites, 3,4-dihydroxyphenylacetic acid and homovanillic acid, progressively increased for 2-3 $\mathrm{h}$ after probe implantation and decreased markedly by $24 \mathrm{~h}$ later. Furthermore, in contrast to DA, the DA metabolites decreased even further after the second probe insertion. Amphetamine-stimulated DA release was also greatly attenuated following the second probe insertion, relative to the first probe insertion. Two probe insertions had only modest effects on
\end{abstract}

the concentration of 5-hydroxyindoleacetic acid in dialysate, relative to the DA metabolites. It is suggested the effects of two probe insertions on DA metabolism and amphetaminestimulated DA release described here are indicative of probeinduced damage to the nigrostriatal DA system. If this is the case, multiple probe insertions may not provide a feasible strategy for within-subjects design dialysis experiments over extended periods of time, at least in the DA system of small animals. It is suggested further that a stable basal concentration of DA in dialysate may be an especially poor indicator of the integrity of the dopaminergic input to the striatum. Key Words: Amphetamine-Caudate nucleus-Dopamine-Serotonin-Microdialysis methods--Striatum. Camp D. M. and Robinson T. E. On the use of multiple probe insertions at the same site for repeated intracerebral microdialysis experiments in the nigrostriatal dopamine system of rats. J. Neurochem 58, 1706-1715 (1992).
In vivo microdialysis is becoming an increasingly popular sampling technique for studying the neurochemistry of the extracellular environment in awake animals (Ungerstedt, 1984). The technique is relatively new, however, and its strengths and limitations are still a topic of investigation (Westerink et al., 1987). For example, it would be advantageous if microdialysis could be used for repeated sampling over long periods of time (days to weeks), because this would permit the use of within-subjects design experiments. There have been a few studies on the use of continuous intracerebral dialysis sampling for 7-10 days. These have not been very promising because of a progressive decline in the amount of neurotransmitter recovered in dialysate (Imperato and Di Chiara, 1984, 1985; Korf and Venema, 1985; Westerink and Tuinte, 1986; Reiriz et al.,
1989), which has been attributed to impeded diffusion caused by the formation of a glial barrier around the dialysis membrane (Westerink and Tuinte, 1986; Benveniste and Diemer, 1987; cf., Robinson and Camp, $1991 b$ ).

There are, however, alternatives to continuous sampling. For example, it may be less disruptive to use multiple insertions of a dialysis probe into the brain, at different points in time, via the same guide cannula. This approach has been used in experiments involving push-pull perfusion (Myers, 1977). We know of only two reports involving multiple probe insertions for within-subjects design dialysis experiments in the mesostriatal dopamine (DA) system (Kalivas and Duffy, 1990; Pettit and Justice, 1991), but neither of these was intended specifically to characterize the procedure.
Received September 11, 1991 ; accepted October 8, 1991.

Address correspondence and reprint requests to Dr. T. E. Robinson at Neuroscience Laboratory Building, The University of Michigan, 1103 E. Huron St., Ann Arbor, MI 48109, U.S.A.
Abbreviations used: ANOVA, analysis of variance; DA, dopamine; DOPAC, 3,4-dihydroxyphenylacetic acid; 5-HIAA, 5-hydroxyindoleacetic acid; HVA, homovanillic acid; 6-OHDA, 6-hydroxydopamine; TTX, tetrodotoxin. 
Thus, the purpose of experiments reported here was to characterize the effects of one or two insertions of a small diameter, concentric-style dialysis probe into the same site in the neostriatum on indices of DA and serotonin function.

\section{MATERIALS AND METHODS}

\section{Subjects, surgical procedures, and probe implantation}

Holtzman rats weighing $250-350 \mathrm{~g}$ were anesthetized with sodium pentobarbital, supplemented with methoxyflurane; unilateral (experiment 1) or bilateral (experiments 2 and 3) 21-gauge stainless steel guide cannulae $(8 \mathrm{~mm}$ long) were placed on the dural surface above the corpus of the striatum, and fixed to the skull with dental cement, using standard stereotaxic techniques. A stylet (26-gauge stainless steel wire) protruded $1 \mathrm{~mm}$ beyond the tip of the cannula to maintain patency. Animals were allowed to recover for at least 1 week before dialysis experiments began. During this time and between dialysis test sessions, animals were housed singly in hanging wire cages in a light-controlled room (lights on at 0600 , off at 2000), with free access to food and water.

Before a dialysis probe (described below) was lowered into the striatum via the guide cannula, it was connected to a liquid swivel (Instech Labs) and the perfusion solution was pumped through the probe at $1.5 \mu \mathrm{l} / \mathrm{min}$. In the first experiment, the perfusion solution, which was always filter-sterilized, consisted of $128.3 \mathrm{~m} M \mathrm{NaCl}, 2.68 \mathrm{~m} M \mathrm{KCl}, 1.35 \mathrm{~m} M$ $\mathrm{CaCl}_{2}$, and $2.0 \mathrm{mM} \mathrm{MgCl}_{2}, \mathrm{pH}$ 7.3. In the second and third experiments, the perfusion solution contained $145 \mathrm{mM} \mathrm{NaCl}$, $2.7 \mathrm{~m} M \mathrm{KCl}, 1.2 \mathrm{~m} M \mathrm{CaCl}_{2}, 1.0 \mathrm{~m} M \mathrm{MgCl}_{2}$, and $0.2 \mathrm{~m} M$ ascorbic acid, pH 7.3 (Moghaddam and Bunney, 1989). Immediately prior to probe implantation, each rat was lightly anesthetized with methoxyflurane and a dialysis probe manually lowered into the striatum via the guide cannula and fixed in place. An elastic harness was fitted about the torso of each animal, and this was connected to the swivel via a flexible tether constructed from model airplane control cable. The animals were then placed into test chambers, with food and water freely available. Dialysate was collected into $400-$ $\mu \mathrm{l}$ polyethylene tubes mounted on the tether approximately $30-40 \mathrm{~cm}$ above the animal.

Experiment 1: Effects of continuous dialysis over 24 $h$ after probe insertion on the basal concentrations of DA, 3,4-dihydroxyphenylacetic acid (DOPAC), homovanillic acid (HVA), and 5-hydroxyindoleacetic acid (5-HIAA) in dialysate

Both male $(n=5)$ and female $(n=8)$ rats were used in this experiment. Beginning no longer than $5 \mathrm{~min}$ after a probe was lowered into the striatum, nine consecutive 20-min samples of dialysate were collected, with the perfusion solution pumped at a rate of $1.5 \mu \mathrm{l} / \mathrm{min}$. Following collection of the last sample, the pump was turned down to $0.3 \mu \mathrm{l} / \mathrm{min}$, and the animals were then left in the test chambers overnight with their probe in place.

At approximately 0900, the day after probe implantation, the pump was again set at $1.5 \mu \mathrm{l} / \mathrm{min}$, and after $15-30 \mathrm{~min}$, five 20-min baseline samples were collected. After the last sample was obtained, the probe was removed, the stylet placed back into the guide cannula, and animals returned to their home cage; 3-5 days later, animals were given an overdose of chloral hydrate, perfused through the heart with $0.9 \%$ sa- line, followed by $10 \%$ formalin in saline, and the brains were removed for histological analysis. The brains were cut using a frozen technique, and $40-\mu \mathrm{m}$ horizontal sections were stained with cresyl violet.

In addition, to determine if basal DA release was tetrodotoxin (TTX)-sensitive, the normal perfusion solution was replaced with one containing $0.5 \mu M$ TTX in a subset of animals $(n=7)$, after collecting normal basal samples on each day of dialysis.

\section{Experiment 2: Effects of two probe insertions separated by 1 week on the basal concentrations of} DA, DOPAC, HVA, and 5-HIAA in dialysate

First probe insertion. Male rats were prepared with bilateral guide cannulae as described above. At least 5 days following surgery, a dialysis probe was lowered into one $(n=2)$ or both $(n=4)$ striata via the guide cannulae and fixed in place. Beginning no longer than $5 \mathrm{~min}$ after the probe was lowered into the striatum, nine consecutive 20 -min samples of dialysate were collected, with the perfusion solution pumped at $1.5 \mu \mathrm{l} / \mathrm{min}$. The pump was then turned down to $0.3 \mu \mathrm{l} / \mathrm{min}$ and the animals left in the test chambers overnight with the probes in place. At approximately 0900 the next day, the pump was again set at $1.5 \mu \mathrm{l} / \mathrm{min}$, and after $15-30 \mathrm{~min}$, at least three 20 -min baseline samples were collected. After the last sample was obtained, the probes were removed, the stylets placed back into the guide cannulae, and animals returned to their home cage.

Second probe insertion. One week later, a second dialysis experiment was performed on these same animals. In this experiment, dialysis probes were again lowered into one or both striata via the bilaterally positioned guide cannulae, and the experimental protocol was exactly as described following the first probe insertion. At the end of this experiment, all animals received an overdose of sodium pentobarbital, and after the probes were removed, the animals were perfused and the brains obtained for histological analysis, as in experiment 1 .

\section{Experiment 3: Effects of two probe insertions} separated by 1 week on basal and amphetamineinduced changes in DA, DOPAC, HVA, and 5-HIAA

First probe insertion. Male rats were prepared with bilateral guide cannulae as described above. On the day before the first dialysis test session, a dialysis probe was lowered into the striatum in one hemisphere via one of the guide cannulae (right and left sides balanced within each group) and fixed in place. Animals were then placed into the test chambers and left there overnight, undisturbed, with the perfusion fluid pumped at $0.3 \mu \mathrm{l} / \mathrm{min}$.

The first dialysis test session began $16-20 \mathrm{~h}$ after a probe was lowered. The pump speed was increased to $1.5 \mu \mathrm{l} / \mathrm{min}$, and after 15-30 min, baseline samples of dialysate were collected. After at least three 20-min baseline samples were collected, animals received intraperitoneally either $2.0 \mathrm{mg} / \mathrm{kg}$ $d$-amphetamine sulfate (weight of the salt; $n=14$ ) or $0.9 \%$ saline $(1 \mathrm{ml} / \mathrm{kg} ; \mathrm{n}=6)$, and then sample collection continued for $200 \mathrm{~min}$. After this, the probe was removed, a stylet placed into the guide cannula, and the animals returned to their home cage. Half the animals given amphetamine during this test session received three additional injections of amphetamine $(5.0 \mathrm{mg} / \mathrm{kg})$ in their home cage, on each of the next 3 days (group AMPH-4), and all other animals received saline.

Second probe insertion. One week after the first dialysis test session, a second dialysis experiment was performed on 
these same animals. In this test session, dialysis probes were lowered into the striatum in both hemispheres via the bilaterally positioned guide cannulae. The experimental protocol was exactly as described following the first probe insertion, except all animals received an injection of $2.0 \mathrm{mg} / \mathrm{kg} \mathrm{am}$ phetamine following collection of baseline samples.

Thus, some animals received amphetamine for the first time during the second dialysis test session, i.e., the saline pretreatment group (SAL), others received amphetamine for the second time during the second dialysis test session (AMPH-1 group), and yet others received amphetamine for the fifth time during the second dialysis test session (AMPH4 group). In addition, in all animals a probe was lowered into one striatum for the first time during the second test session (the "naive" side) and the other striatum received a probe for the second time (the "used" side). Finally, one additional control group of rats underwent dialysis only once (control; $\mathrm{n}=6$ ), and had a dialysis probe lowered into each striatum. This control group received exactly the same treatment as saline-pretreated animals, except no probe was lowered during the first dialysis test session (i.e., animals were just placed into the test chamber).

At the end of the second test session, all animals received an overdose of sodium pentobarbital, and after the probes were removed, the brains were obtained for histological analysis, as in experiment 1 .

\section{Microdialysis probe}

A removable version of a concentric-style microdialysis probe described previously was used (Robinson and Whishaw, 1988; Robinson and Camp, 1991a). Briefly, the dialysis membrane consisted of a 4-mm length of regenerated cellulose hollow dialysis fiber with an outside diameter of 230$250 \mu \mathrm{m}$ and a molecular weight cutoff of 6,000 . The inlet line consisted of polyethylene 20 tubing and the outlet line of fused silica capillary tubing.

Each probe was tested in vitro at $37^{\circ} \mathrm{C}$ to determine its ability to recover known concentrations of DA, DOPAC, HVA, and 5-HIAA. Recovery was determined both prior to implantation and again following removal of the probe from the brain. Probes were used for one or two experiments, and between experiments probe tips were kept submersed in a $1.5 \%$ formalin solution. Prior to the first time a probe was used (preinsertion), the average relative recovery values for DA, DOPAC, HVA, and 5-HIAA were $25.55 \pm 1.19 \%, 25.23$ $\pm 0.95 \%, 23.60 \pm 0.85 \%$, and $25.58 \pm 1.06 \%$, respectively. Following removal after their first use, the recovery values were $26.02 \pm 0.86 \%, 22.38 \pm 0.80 \%, 22.28 \pm 0.82 \%$, and $24.39 \pm 0.93 \%$, respectively, but the change was statistically significant only for DOPAC. After probes were used a second time, recovery again declined somewhat, to $24.13 \pm 0.77 \%$, $20.91 \pm 0.71 \%, 19.96 \pm 0.67 \%$, and $21.63 \pm 0.94 \%$ for $D A$ DOPAC, HVA, and 5-HIAA, respectively, and this decrease was statistically significant for DOPAC, HVA, and 5-HIAA, relative to the preinsertion values. In this experiment (i.e., probes used in experiment 1 ), the change in DA recovery was not statistically significant, but in other unpublished experiments a comparable small decrease in DA recovery was statistically significant.

\section{Assay of dialysate}

The concentrations of DA, DOPAC, HVA, and 5-HIAA in dialysate were determined with HPLC and oxidative-reductive electrochemical detection, as described previously (Robinson, 1990; Robinson and Camp, 1991a). The amount of each compound in dialysate was determined by comparison with the peak heights of standards run with each experiment. In addition, the purity of DA in dialysate was determined by (1) comparing the retention time of DA in the standard to that in dialysate, (2) sometimes spiking the sample with DA, and (3) periodically using oxidation at a high potential $(+400 \mathrm{mV})$, followed by series reduction at two different points $(-110$ and $-250 \mathrm{mV})$ on the reduction current/ voltage curve for DA (Matson et al., 1984). The series reduction ratios for DA obtained from striatal dialysate did not differ from those obtained for a standard solution of DA in four such experiments. The minimum detection level for DA was about $1 \mathrm{fmol} / \mathrm{min}$, using a criterion signal:noise ratio of $3: 1$.

\section{Data analysis}

Data are included for only those animals in which the probe was placed correctly in the corpus of the neostriatum (see Fig. 4 in Robinson and Whishaw, 1988). In addition, data from one animal in experiment 2 and four animals in experiment 3 were excluded, because histological analysis revealed extensive tissue damage surrounding the probe track.

The concentration of each compound is expressed in femtomoles per minute, corrected for probe recovery. When probes were reused, the appropriate recovery values were used (see above). It is important to note that the corrected values do not reflect the actual extracellular concentrations of these compounds, because in vitro recovery procedures do not take into account diffusion kinetics in a complex medium, such as brain (Benveniste and Hansen, 1991; Morrison et al., 1991). Recovery values were used here primarily to screen for probes with poor recovery characteristics and to control for experimental error due to variation in probe construction.

Statistical analyses were performed using a Macintosh II computer with Systat 5.0 and StatView II (BrainPower) software. Time-course data were analyzed using analyses of variance (ANOVAs) with one or two repeated measures. Comparisons of individual time points were made using either Student's two-tailed $t$ tests for planned comparisons, or Fisher's least significant difference (LSD) test for pairwise comparisons [all two-tailed probabilities unless otherwise noted (Winer, 1971)]. In addition, selected correlational analyses (Pearson's $r$ ) were performed.

\section{RESULTS}

Experiment 1: Effects of continuous dialysis over 24 $h$ on the basal concentrations of DA, HVA, DOPAC, and 5-HIAA in dialysate

There was no difference between male and female rats and, therefore, their data were pooled for presentation in Fig. 1. Figure 1 shows the basal concentrations of DA and the three monoamine metabolites in dialysate during the first $(0-3 \mathrm{~h})$ and second $(24 \mathrm{~h}$ later) day following probe insertion. The concentration of $\mathrm{DA}$ in dialysate was elevated during the first 20-min interval after insertion of the dialysis probe (Fig. 1A). However, DA levels stabilized within $40 \mathrm{~min}$ and remained stable for the next $3 \mathrm{~h}$. The next day, $24 \mathrm{~h}$ after probe insertion, the average concentration of DA in dialysate was lower than the 3-h value the day before, but this difference was not statistically significant (see Fig. 1A).

In contrast to DA, the concentrations of DOPAC, 
FIG. 1. The mean ( \pm SEM) concentrations ( $\mathrm{fmol} / \mathrm{min}$ ) of DA (A), DOPAC (B), HVA (C), and 5-HIAA (D) in dialysate for nine consecutive 20-min intervals immediately following insertion of a dialysis probe into the striatum (0-3 $h ; n=13$ ), and for five 20-min intervals beginning $24 \mathrm{~h}$ later. All four compounds showed highly significant changes over time during the first $3 \mathrm{~h}$ after probe insertion (one-way ANOVAs with repeated measures, all $p$ values $<0.0001$ ). A: Comparisons of individual points in time $0-3 \mathrm{~h}$ following the first probe insertion showed that DA stabilized by the second 20 -min sample (i.e., the first 20-min interval was the only point that differed from any other point in time; Fisher PLSD test, $p<0.05$ ). The average basal concentration of DA in dialysate was lower $24 \mathrm{~h}$ later in all animals that were tested

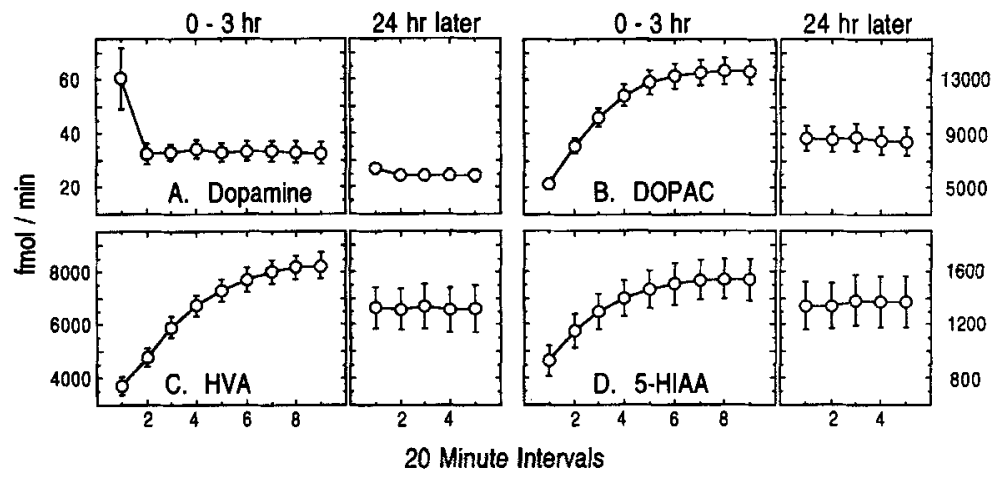
on both days, except one $\left(n=8 ; \chi^{2}\right.$ test, $\chi^{2}=4.5$, $p<0.05$ ), although in this experiment the decrease in DA was not statistically significant with a paired $t$ test comparing the ninth 20 -min interval on the first day with the average of the five 20-min samples collected on the second day $(t=1.42, p=0.20)$. B, C, and $D$ : Comparisons of individual points in time showed that metabolite concentrations reached asymptote $100-140$ min following probe insertion (Fisher PLSD tests, $p<0.05$ ). By $24 \mathrm{~h}$ later, DA metabolite concentrations declined significantly, relative to the 3-h values (paired $t$ tests; DOPAC: $t=9.50, p<0.0001$; HVA: $t=3.85, p=0.005)$, but the small decrease in 5-HIAA was not significant $(t=0.96)$.

HVA, and 5-HIAA in dialysate steadily increased over the first $2 \mathrm{~h}$ following insertion of the probe, but reached asymptote by $2-3 \mathrm{~h}$. However, $24 \mathrm{~h}$ later, there was a decrease in the metabolite concentrations, relative to the 3-h value, although this was statistically significant only for DOPAC and HVA. The increase in DA metabolite concentrations over the first $3 \mathrm{~h}$ after probe insertion was not due to the effects of brief anesthesia at the time of probe implantation, because in an independent group of animals $(n=4)$ that were not anesthetized during the probe implantation procedure, a similar increase in DA metabolite concentrations was seen (data not shown). For example, the percent increase in DOPAC between the first and ninth sample was $171 \pm 19 \%$ in animals that were briefly anesthetized and $191 \pm 17 \%$ in animals that were not briefly anesthetized.

The addition of TTX to the perfusion solution produced a significant decrease in the concentration of DA in dialysate, but TTX reduced DA to a significantly greater extent $24 \mathrm{~h}$ after probe implantation (to 18.63 $\pm 5.24 \%$ of baseline by the end of the infusion period) than on the same day as probe implantation (to 43.72 $\pm 9.64 \%$ of baseline), consistent with previous reports (Westerink and de Vries, 1988; Santiago and Westerink, 1990).

\section{Experiment 2: Effects of two probe insertions} separated by 1 week on the basal concentrations of DA, DOPAC, HVA, and 5-HIAA in dialysate

Figure 2 shows the basal concentrations of DA, DOPAC, HVA, and 5-HIAA in dialysate $3 \mathrm{~h}$ and $24 \mathrm{~h}$ after the first probe insertion, and then again $3 \mathrm{~h}$ and $24 \mathrm{~h}$ after the second probe insertion 1 week later. In this experiment, there was a statistically significant decrease in the basal concentration of DA over $24 \mathrm{~h}$ following both the first and second probe insertions (i.e., the 3- and 24-h values differ in both cases; see Fig. 2A).
There was no effect, however, of probe insertion. The basal concentrations of DA 3 and $24 \mathrm{~h}$ after the second probe insertion were the same as seen 3 and $24 \mathrm{~h}$ following the first probe insertion, respectively.

In contrast to DA, the basal concentrations of the DA metabolites were not stable between two probe insertions. Basal DOPAC and HVA (Fig. 2B and C) decreased significantly between 3 and $24 \mathrm{~h}$ after the first probe insertion. Three hours after the second probe insertion, the basal concentrations of DOPAC and HVA were the same as seen $24 \mathrm{~h}$ after the first probe insertion, but over the next $24 \mathrm{~h}$ DOPAC and HVA decreased even further relative to all other points in time. The basal concentration of 5-HIAA was influenced by time after probe implantation and probe insertion in a fashion similar to that of the DA metabolites (Fig. 2D), but the magnitude of the change in 5-HIAA was much less pronounced. For example, DOPAC decreased by $74.8 \pm 4.1 \%$ between $3 \mathrm{~h}$ following the first probe insertion and $24 \mathrm{~h}$ following the second probe insertion, whereas 5-HIAA decreased by only $45.3 \pm 4.8 \%$ over this same period of time.

\section{Experiment 3: Effects of two probe insertions} separated by 1 week on basal DA, DOPAC, HVA, and 5-HIAA, and on amphetamine-stimulated DA release

Basal values. Figure 3 shows the basal concentrations of DA, DOPAC, HVA, and 5-HIAA in dialysate measured $16-20 \mathrm{~h}$ after probe implantation in three groups. (1) In the first group, probes were lowered twice via the same guide cannula, with probe insertions separated by 1 week (first insertion versus second insertion in Fig. 3). (2) The second group consisted of the same animals as in (1), but the values are from probes that were lowered into the contralateral naive striatum only once, during the second test session (naive side in Fig. 3). (3) The third group consisted of an independent 


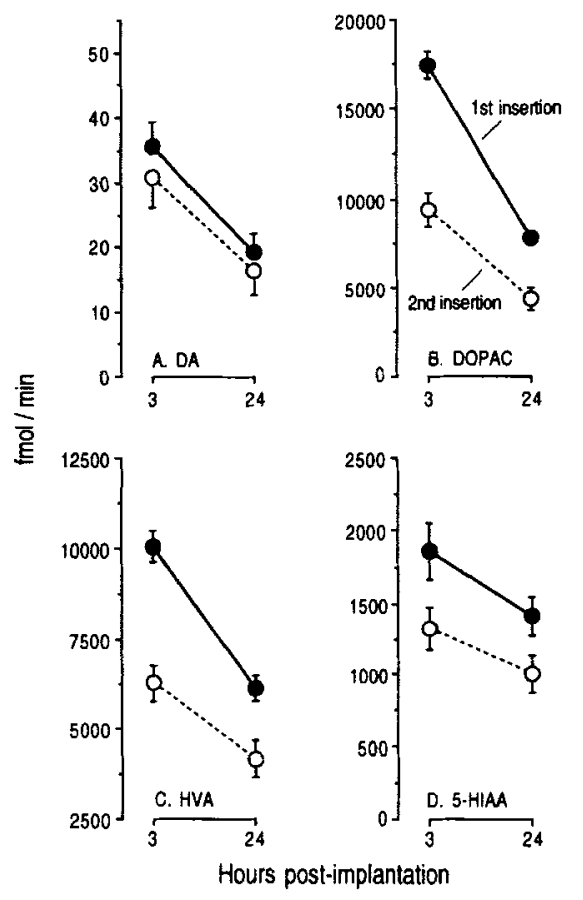

FIG. 2. The mean ( \pm SEM) basal concentrations of DA (A), DOPAC (B), HVA (C), and 5-HIAA (D) in dialysate: (1) $3 \mathrm{~h}$ and then again $24 \mathrm{~h}$ after the first probe insertion (O), and (2) $3 \mathrm{~h}$ and $24 \mathrm{~h}$ after the second probe insertion, 1 week later (O). A: A two-way ANOVA with repeated measures on both factors (hours post implantation by insertion) showed that there was a significant decrease in DA between 3 and $24 \mathrm{~h}$ after probe insertion, and this occurred after both the first and second probe insertions $(F=77.95, p<0.0001)$. There was, however, no effect of probe insertion independent of hours post implantation $(F=0.7, p=0.44$; interaction, $F=0.27$, $p=0.62$ ). B: A two-way ANOVA resulted in a significant effect of hours post implantation $(F=335, p<0.0001)$, a significant effect of probe insertion $(F=32.3, p=0.001)$, and a significant hours by insertion interaction $(F=29.42, p=0.001)$. Subsequent paired $t$ tests showed that all points differed significantly $(p$ values $<0.006$ ), except $24 \mathrm{~h}$ after the first insertion versus $3 \mathrm{~h}$ after the second insertion. $C$ : The results were qualitatively the same as for DOPAC (effect of hours post implantation, $F=150.6, p<0.0001$; effect of probe insertion, $F=57.6, \rho<0.0001$; interaction, $F=15.83$, $p=0.005)$. Paired comparisons were as for DOPAC ( $p$ values $<0.004)$. D: Effect of hours post implantation, $F=42.42, \rho<$ 0.0001 ; effect of probe insertion, $F=21.75, p=0.002$; interaction nonsignificant, $F=0.68, p=0.44$. In summary, the concentration of DA in dialysate was stable between two probe insertions separated by 1 week, but those of the monoamine metabolites were not.

group of control animals, in which probes were lowered bilaterally only once, during the second test session (controls in Fig. 3).

As in experiment 2, there was no difference in the basal concentration of DA in dialysate measured 16$20 \mathrm{~h}$ following the first versus second probe insertion. There was also no difference in the DA values between the control group and any other group, although DA in the naive striatum was slightly, but significantly, lower than in the used hemisphere during the first test session (i.e., first insertion in Fig. 3). Presumably, this latter effect reflects a chance sampling difference, be- cause the histological analysis showed that the probe placements were completely overlapping between the two sides.

In contrast to DA, there was a marked decrease in basal DOPAC between the first and second probe insertions, confirming experiment 2 (Fig. 3). The decrease in DOPAC between the first and second probe insertions was also apparent relative to both the contralateral naive striatum and values obtained in control animals. HVA was also significantly lower during the second probe insertion, relative to the first. However, unlike DOPAC, no other comparisons were significant. In contrast to the DA metabolites, there was no difference in the concentration of 5-HIAA in dialysate between the first and second probe insertions, and none of the other comparisons was significant either (Fig. 3).

Amphetamine-stimulated $D A$ release. Figure 4 shows the effect of amphetamine on the concentration of DA in dialysate, as a function of probe insertion in both amphetamine- and saline-pretreated groups. During the first test session, amphetamine produced a large increase in the concentration of DA in dialysate, which peaked within 20-40 min (AMPH-1st in Fig. 4). However, during the second test session, the response to amphetamine was significantly attenuated, relative to the first test session (AMPH-1st versus AMPH-2nd in Fig. 4). The response of saline-pretreated animals to amphetamine during the second test session was also

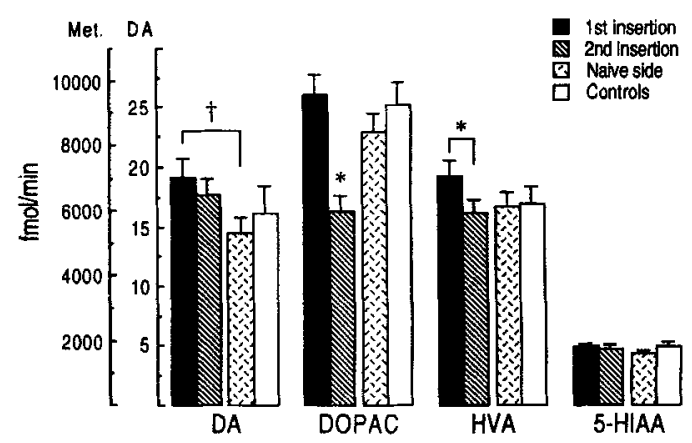

FIG. 3. The mean (+ SEM) basal concentrations ( $\mathrm{fmol} / \mathrm{min}$ ) of $\mathrm{DA}$, DOPAC, HVA, and 5-HIAA in dialysate measured 16-20 $\mathrm{h}$ following the initial probe insertion (first insertion, $n=20$ ), and then again following the second insertion of a probe via the same guide cannula 1 week later (second insertion, $n=20$; the amphetamine- and saline-pretreatment groups did not differ on any of the basal measures and, therefore, were pooled for analysis here; see experiment 3 methods). In addition, "naive side" refers to values obtained from a probe lowered for the first time during the second test session, via a guide cannula located in the hemisphere contralateral to that used during the first test session $(n=20)$. "Controls" refers to values obtained with an independent group of animals (n $=12$ probes, six rats) in which probes were lowered only once, bilaterally. During the first test session, these latter animals received exactly the same treatment as saline-pretreated animals (i.e., briefly anesthetized, saline injection), but no probe was lowered. The basal value for each individual animal consisted of the average of three successive 20-min baseline samples. Groups connected by a line above the bars differed significantly, based on unpaired or paired $t$ tests $\left(t p=0.01 ;{ }^{*} p<0.006\right.$, two-tailed probabilities). For DOPAC, the asterisk indicates that this group differed significantly from all other groups. No other comparisons were significant. 


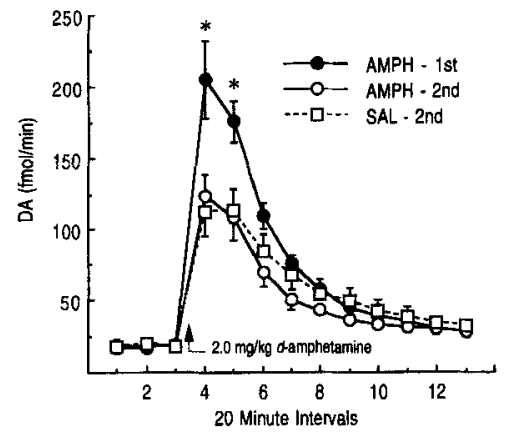

FIG. 4. The effect of a challenge injection of $2.0 \mathrm{mg} / \mathrm{kg} d$-amphetamine sulfate on the mean ( \pm SEM) concentration of DA in dialysate $(\mathrm{fmol} / \mathrm{min})$. The first three $20-\mathrm{min}$ samples were obtained prior to amphetamine administration, which was given where indicated by the arrow. Data are shown: (1) from animals that received amphetamine during the first dialysis test session (AMPH-1st, $\bullet$, $n=14 ;$ AMPH-4 and AMPH-1 groups pooled; see Materials and Methods); (2) from the same animals when they again received amphetamine 1 week later, the day after a probe was reinserted via the same guide cannula (AMPH-2nd, $O, n=14$ ); and (3) from an independent group of animals that underwent dialysis during the first test session, but received saline at that time; shown here is their response when given amphetamine for the first time during the second test session (SAL-2nd, $\square, n=6$ ). Amphetamine-stimulated DA release was attenuated significantly during the second dialysis test session in both amphetamine- and saline-pretreated groups relative to the first test session. AMPH-1 st versus AMPH2nd: two-way ANOVA with repeated measures on both factors; effect of probe insertion, $F=10.1, p=0.007$; effect of time after amphetamine, $F=74.8, p<0.0001$; insertion by time interaction, $F=6.02, p<0.0001$. AMPH-1 st versus SAL-2nd: two-way ANOVA with one repeated measure (time); effect of group, $F=2.43, p$ $=0.14$; effect of time, $F=33.9, p<0.0001$; interaction, $F=4.38$, $p<0.0001$. AMPH-2nd versus SAL-2nd: only the effect of time was significant (the group and interaction $F$ values $<0.7$ ). Asterisks indicate the individual points that differed between AMPH-1st values and the comparable values for both the AMPH-2nd and SAL-2nd groups $(p<0.05)$.

significantly diminished relative to animals that received amphetamine during the first test session (AMPH-1 st versus SAL-2nd in Fig. 4).

A comparison of the effect of the amphetamine challenge during the second test session in the naive versus used striatum produced similar results. That is, during the second test session, the peak concentration of DA following the amphetamine challenge was significantly higher in both the naive striatum and control animals than in the used striatum (naive side $=1,280$ $\pm 142 \%$ of baseline, controls $=1,115 \pm 106 \%$ of baseline, and the used striatum $=745 \pm 54 \%$ of baseline). However, the animals that received four previous injections of amphetamine were also analyzed separately (AMPH-4; see Materials and Methods). Figure 5 shows that amphetamine produced a significantly greater increase in DA in the naive striatum of these animals than in the naive striatum of control animals (salinepretreated and control groups pooled; Fig. 5). This latter finding is consistent with previous reports of sensitization following repeated administration of amphetamine (e.g., Robinson et al., 1988).

\section{Correlational analyses}

Correlational analyses were performed on the data from experiment 3 to determine the relationship between the basal concentrations of DA, DOPAC, HVA, and 5-HIAA in dialysate following the first versus second probe insertion. There was no statistically significant correlation in basal DA between the two probe insertions $(r=0.221)$. In contrast, the basal concentrations of DOPAC, HVA, and 5-HIAA measured following the first probe insertion were all positively and significantly correlated with the respective values obtained following the second probe insertion ( $r$ values of $0.60,0.69$, and 0.74 , respectively; $p$ values $<0.01$ ). Correlational analyses of the percent change in basal DA, basal DOPAC, and peak amphetamine-stimulated DA release between the two probe insertions were especially interesting. The magnitude of the decline in basal DOPAC between the two probe insertions was significantly and positively correlated with the magnitude of the decline in peak amphetamine-stimulated DA release $(r=0.64, p<0.001)$. However, the change in basal DA was not significantly correlated with the change in either basal DOPAC $(r=-0.23)$ or amphetamine-stimulated DA release $(r=0.00)$. Therefore, the change in basal DOPAC, but not the change in basal DA, predicted the change in amphetamine-stimulated DA release between two probe insertions.

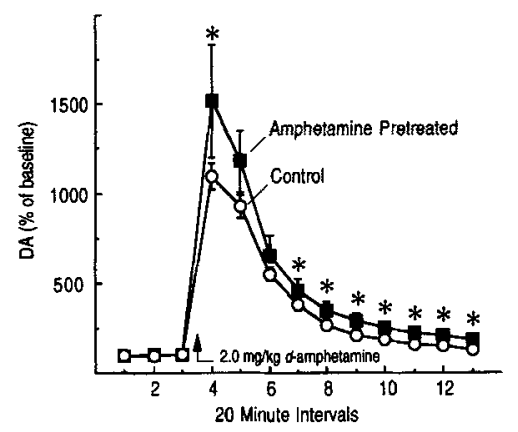

FIG. 5. The effect of $2.0 \mathrm{mg} / \mathrm{kg}$ amphetamine given during the second dialysis test session on the mean ( \pm SEM) concentration of DA in dialysate obtained from the naive striatum (i.e., the side that did not receive a probe during the first dialysis test session). The first three intervals represent basal DA, and each subsequent interval was obtained following the amphetamine challenge (with values expressed as a percentage of the average baseline value). The amphetamine-pretreated group $(\square ; n=7)$ represents that subset of animals given amphetamine during the first dialysis test session, and then three additional injections of amphetamine in their home cage between the first and second test sessions (see text for details). The control group $(0 ; n=18)$ consisted of those animals that received amphetamine for the first time during the second test session (saline-pretreated and control groups pooled). The challenge injection produced a significantly greater increase in DA in the naive striatum of amphetamine-pretreated animals than in the naive striatum of the control group (two-way ANOVA with repeated measures: effect of pretreatment, $F=4.17, p=0.053$; interaction, $F=2.4, p=0.013 ;{ }^{*} p=0.05$, independent $t$ tests, one-tailed probabilities). The sensitization of amphetamine-stimulated DA release was predicted on the basis of previous betweensubjects design studies (e.g., Robinson et al., 1988), which is why one-tailed tests were used. 


\section{DISCUSSION}

The experiments reported here revealed a consistent dissociation in the effects of two insertions of a dialysis probe into the same site on basal DA versus other measures of DA neurotransmission. For example, there was no significant difference in the basal concentration of DA in dialysate between two probe insertions separated by 1 week, whereas the basal concentrations of DOPAC and HVA in dialysate progressively decreased. Like the DA metabolites, the ability of amphetamine to elevate DA in dialysate was greatly attenuated following a second probe insertion, and the magnitude of the decrease in amphetamine-stimulated DA release was positively correlated with the magnitude of the decrease in basal DOPAC. The implications of these findings for the use of within-subjects design dialysis experiments involving multiple probe insertions at the same site are discussed below.

\section{Effects of two probe insertions on the basal concentration of DA and monoamine metabolites in dialysate}

If the effects of insertion and reinsertion of a small diameter, concentric-style dialysis probe on basal DA were considered in isolation, it would appear that the nigrostriatal DA system was not disturbed markedly by the procedures employed here. Not only did basal DA stabilize quickly after each probe insertion, but it remained stable across two insertions of a probe at the same site. Using the concentric-style probe described here, Robinson and Camp (1991b) also found no significant decrease in basal DA over 4 days of continuous dialysis. Thus, measures of basal DA suggest that multiple probe insertions at the same site may provide a reasonable approach for within-subjects design dialysis experiments. On the other hand, there was not a significant correlation in basal DA between two probe insertions separated by 1 week, and the effects of two probe insertions on DA metabolism were not encouraging.

The basal concentrations of the DA metabolites, DOPAC and HVA, progressively increased over the first 2-3 h after probe implantation and then decreased significantly $24 \mathrm{~h}$ later. By $24 \mathrm{~h}$ following the second probe insertion, basal DOPAC and HVA had declined even further. Consistent with this, Robinson and Camp (1991b) reported a progressive decrease in the basal concentration of DA metabolites in dialysate over 4 days of continuous dialysis. It is important to consider, therefore, what a decrease in DA metabolites tells us about the nature of probe-induced alterations in the nigrostriatal DA system. One possibility is that the changes in DOPAC reflect the extent to which the nigrostriatal DA system was compromised by the dialysis procedure. The implication of this, of course, is that DOPAC better reflects the integrity of the dopaminergic input to the striatum than does the basal concentration of DA. Although this may seem counterintuitive at first, such an interpretation is consistent with micro- dialysis studies in which the nigrostriatal DA system was deliberately damaged by use of either 6-hydroxydopamine (6-OHDA) or neurotoxic doses of methamphetamine (Robinson and Whishaw, 1988; Touchet and Bennett, 1989; Abercrombie et al., 1990; Castañeda et al., 1990; for review, see Robinson et al., 1990).

It has been shown that the postmortem tissue concentration of DA in the striatum can be depleted by up to $80 \%$, with no change in the basal concentration of DA in striatal dialysate (Robinson et al., 1990). Following a neurotoxic lesion, the postmortem tissue concentration of DA is known to provide a good index of the magnitude of DA denervation and the extent of cell loss in the substantia nigra (Ranje and Ungerstedt, 1977; Onn et al., 1986). Destruction of 80-95\% of the DA innervation in the striatum produces more variable effects, although after recovery some animals with even this degree of DA denervation still have basal levels of dialysate DA within the control range (Castañeda et al., 1990). Only after a lesion destroys over $95 \%$ of the DA input to the striatum is there a consistent and marked decrease in dialysate DA (Castañeda et al., 1990). The normalization of basal DA in dialysate seen following partial damage to the nigrostriatal DA system is thought to be due to the interaction of many presynaptic compensatory changes in the remaining DA terminals, including an increase in DA synthesis and release and a decrease in DA reuptake (Robinson et al., 1990; Zigmond et al., 1990). Presumably, it is because of these neuroplastic adaptations that the basal concentration of DA in dialysate is such a poor indicator of the integrity of the nigrostriatal DA system.

In contrast to DA, a 6-OHDA lesion produces a decrease in the concentration of DOPAC in dialysate that is proportional to the extent of the lesion (Zhang et al., 1988; Altar and Marien, 1989; Castañeda et al., 1990). The integrity of the nigrostriatal DA input to the striatum may be predicted by basal DOPAC in dialysate, but not by basal DA, because DOPAC largely reflects DA that is metabolized intraneuronally, independent of DA release (Zetterström et al., 1988; Kuczenski and Segal, 1989). This source of DOPAC would be decreased in proportion to the loss of DA terminals following a lesion. In summary, it is suggested that the decrease in basal DOPAC seen between two probe insertions indicates the extent of damage to the striatum, and this was not reflected by a change in basal DA, because damage-evoked compensatory adaptations were sufficient to maintain a normal extracellular concentration of DA (Robinson et al., 1990).

It is also interesting that two probe insertions had a much smaller effect on the basal concentration of the serotonin metabolite, 5-HIAA, than on the DA metabolites. This suggests that the dissociation between DA and its metabolites discussed above was not due to a nonspecific change in the recovery of acids from the extracellular fluid. This is also consistent with the observation that, relative to the DA metabolites, basal 5-HIAA shows only modest changes over 4 days of 
continuous dialysis (Reiriz et al., 1989; Robinson and Camp, 1991b).

\section{Effect of two probe insertions on amphetamine- stimulated DA release}

The effect of two probe insertions on amphetaminestimulated DA release was similar to that on basal DOPAC, i.e., the ability of amphetamine to elevate DA in dialysate was decreased markedly between two probe insertions separated by 1 week. There are several possible explanations for this decreased responsivity to amphetamine. First, high doses of amphetamine are known to be neurotoxic, causing degeneration of striatal DA terminals, DA depletion in postmortem tissue, a reduced number of DA reuptake sites, and decreased tyrosine hydroxylase activity (Seiden and Ricaurte, 1987). It is important to consider, therefore, whether the decrease in amphetamine-stimulated DA release between the two test sessions (and the decrease in DOPAC) could be secondary to amphetamine neurotoxicity. This is unlikely for three reasons. (1) In the present study, a relatively low dose of amphetamine was used, and neurotoxicity has been reported only after much higher doses (Robinson and Becker, 1986). This is not a strong argument, however, because tissue already damaged by probe implantation could be more susceptible to amphetamine neurotoxicity (e.g., Phebus et al., 1991). It is important, therefore, that (2) salinepretreated rats also showed an attenuated response to amphetamine during the second test session, and (3) the response in the contralateral naive striatum, which had a probe lowered into it for the first time during the second dialysis test session, was similar to that seen during the first dialysis test session. These latter two points indicate that it was probe implantation, and not prior experience with amphetamine, that was responsible for the decrease in amphetamine-stimulated DA release.

Another possibility is that often the same dialysis probe was used in different animals, at different times, and although the brain is considered immunologically privileged, proteins remaining on the probe from one animal could have triggered a negative tissue reaction when used in a different animal. To rule this out, approximately half of the animals in the third experiment received newly constructed probes in both the first and second test sessions. Amphetamine-stimulated DA release in these animals was identical to that in animals which had dialysis performed with a previously used probe (data not shown).

A third possible explanation for the decline in amphetamine-stimulated DA release between two test sessions is that it reflects the extent of damage to the nigrostriatal DA system in the same manner as the decrease in basal DOPAC (see above). This idea is supported by the strong positive correlation between the magnitude of the decrease in amphetamine-stimulated DA release and the magnitude of the decrease in basal DOPAC. One problem with this interpretation, how- ever, is that Castañeda et al. (1990) reported that destruction of up to $80 \%$ of the DA input to the striatum with 6-OHDA had no significant effect on amphetamine-stimulated DA release, despite a decrease in basal DOPAC. That is, following a partial 6-OHDA lesion, compensatory adaptations were not only sufficient to normalize basal DA, but amphetamine-stimulated DA release as well. The reason for a difference in the effects of probe implantation versus a 6-OHDA lesion on amphetamine-stimulated DA release is not clear.

One procedural difference is that Castañeda et al. (1990) tested animals approximately 1 month after a 6-OHDA lesion, whereas in the present study animals were retested for amphetamine-stimulated DA release 1 week after the first test session (i.e., 1 week after the damage produced by the initial probe insertion). Perhaps 1 week is not sufficient time for recovery. Another possibility, however, may be related to the fact that probe implantation and 6-OHDA produce a very different type of lesion. The lesion produced by probe implantation is focal and nonspecific. A 6-OHDA lesion, on the other hand, produces a fairly specific and diffuse "pruning" of the entire DA input to the striatum. It is known that this latter treatment results in a marked increase in DA synthesis in the surviving terminals (Zigmond et al., 1984; Wolf et al., 1989), which may play an important role in the ability of the surviving DA neurons to maintain relatively normal levels of amphetamine-stimulated DA release. Perhaps following probe implantation there is not a comparable increase in DA synthesis in the surviving DA terminals, because the focal nature of the injury does not provide a sufficient "signal" for up-regulation of DA synthesis. Thus, following reinsertion of the dialysis probe, the rate of DA synthesis may be sufficient to maintain the basal concentration of DA in dialysate, but not sufficient to meet the demand for increased DA release imposed by a challenge injection of amphetamine.

\section{Conclusions}

In conclusion, these experiments suggest that withinsubjects design studies using repeated probe insertions at the same site in the striatum of rats are probably illadvised, at least with the methods and materials used here. This does not rule out the possibility of repeated dialysis in the same rat, but at different sites, or repeated microdialysis at the same site in larger animals (Kendrick, 1991), and/or in other neurotransmitter systems. Nevertheless, if a within-subjects design is used for microdialysis, it should be incumbent upon the investigator to demonstrate whether the integrity of the neural system under study is maintained between test sessions. The data presented here and by Robinson and Camp (1991b) suggest that a stable basal concentration of a neurotransmitter in dialysate does not constitute sufficient evidence and, in fact, the transmitter itself may be the least sensitive indicator of system integrity. 
Acknowledgment: The research reported here was supported by grant no. 04294 from the National Institute on Drug Abuse. We thank Zoltan Mocsary and Erin Wolfe for their assistance in conducting some of the experiments. Portions of the data reported here were presented previously in abstract form (Camp and Robinson, 1989, 1990) and in an edited book chapter (Robinson and Camp, 1991).

\section{REFERENCES}

Abercrombie E. D., Bonatz A. E., and Zigmond M. J. (1990) Effects of $l$-Dopa on extracellular dopamine in striatum of normal and 6-hydroxydopamine-treated rats. Brain Res. 525, 36-44.

Altar C. A. and Marien M. R. (1989) Preservation of dopamine release in the denervated striatum. Neurosci. Lett. 96, 329-334.

Benveniste H. and Diemer N. H. (1987) Cellular reactions to implantation of a microdialysis tube in the rat hippocampus. Acta Neuropathol. (Berl). 74, 234-238.

Benveniste H. and Hansen A. J. (1991) Practical aspects of using microdialysis for determination of brain interstitial concentrations, in Microdialysis in the Neurosciences (Robinson T. E. and Justice J. B. Jr., eds), pp. 81-100. Elsevier, Amsterdam.

Camp D. M. and Robinson T. E. (1989) A method for repeated intracerebral microdialysis. Soc. Neurosci. Abstr. 15, 559.

Camp D. M. and Robinson T. E. (1990) Repeated intracerebral microdialysis for the measurement of stimulated dopamine release. Soc. Neurosci. Abstr. 16, 130.

Castañeda E., Whishaw I. Q., and Robinson T. E. (1990) Changes in striatal dopamine neurotransmission assessed with microdialysis following recovery from a bilateral 6-OHDA lesion: variation as a function of lesion size. J. Neurosci. 10, 1847-1854.

Imperato A. and Di Chiara G. (1984) Trans-striatal dialysis coupled to reverse phase high performance liquid chromatography with electrochemical detection: a new method for the study of the in vivo release of endogenous dopamine and metabolites. $J$. Neurosci. 4, 966-977.

Imperato A. and Di Chiara G. (1985) Dopamine release and metabolism in awake rats after systemic neuroleptics as studied by trans-striatal dialysis. J. Neurosci. 5, 297-306.

Kalivas P. W. and Duffy P. (1990) Effect of acute and daily cocaine treatment on extracellular dopamine in the nucleus accumbens. Synapse 5, 48-58.

Kendrick K. M. (1991) Microdialysis in large unrestrained animals: neuroendocrine and behavioral studies of acetylcholine, amino acid, monoamine and neuropeptide release in the sheep, in $\mathrm{Mi}$ crodialysis in the Neurosciences (Robinson T. E. and Justice J. B. Jr., eds), pp. 327-348. Elsevier, Amsterdam.

Korf J. and Venema K. (1985) Amino acids in rat striatal dialysates: methodological aspects and changes after electroconvulsive shock. $J$. Neurochem. 45, 1341-1348.

Kuczenski R. and Segal D. (1989) Concomitant characterization of behavioral and striatal neurotransmitter response to amphetamine using in vivo microdialysis. $J$. Neurosci. 9, 2051-2065.

Matson W. R., Langlais P., Volicer L., Gamache P. H., Bird E., and Mark K. A. (1984) n-Electrode three-dimensional liquid chromatography with electrochemical detection for determination of neurotransmitters. Clin. Chem. 30, 1477-1488.

Moghaddam B. and Bunney B. S. (1989) Ionic composition of microdialysis perfusing solution alters the pharmacological responsiveness and basal outflow of striatal dopamine. $J$. Neurochem. 53, 652-654.

Morrison P. F., Bungay P. M., Hsiao J. K., Mefford I. N., Dykstra K. H., and Dedrick R. L. (1991) Quantitative microdialysis, in Microdialysis in the Neurosciences (Robinson T. E. and Justice J. B. Jr., eds), pp. 47-80. Elsevier, Amsterdam.

Myers R. D. (1977) Chronic methods: intraventricular infusion, cerebrospinal fluid sampling and push-pull perfusion, in Methods in Psychobiology (Myers R. D., ed), pp. 281-315. Academic Press, New York.

Onn S. P., Berger T. W., Stricker E. M., and Zigmond M. J. (1986)
Effects of intraventricular 6-hydroxydopamine on the dopaminergic innervation of striatum: histochemical and neurochemical analysis. Brain Res. 376, 8-19.

Pettit H. O. and Justice J. B. Jr. (1991) Effect of dose on cocaine selfadministration behavior and dopamine levels in the nucleus accumbens. Brain Res. 539, 94-102.

Phebus L. A., Mincy R. E., and Clemens J. A. (1991) Microdialysis perfusion accelerates striatal ischemic damage. Curr. Separations $10,114$.

Ranje C. and Ungerstedt U. (1977) High correlations between number of dopamine cells, dopamine levels and motor performance. Brain Res. 134, 83-93.

Reiriz J., Mena M. A., Bazan E., Muradas V., Lerma J., Delgado J. M., and De Yebenes J. G. (1989) Temporal profile of levels of monoamines and their metabolites in striata of rats implanted with dialysis tubes. $J$. Neurochem. 53, 789-792.

Robinson T. E. (1990) Coulochem Applications: Microdialysates. ESA Co., Bedford, Massachusetts.

Robinson T. E. and Becker J. B. (1986) Enduring changes in brain and behavior produced by chronic amphetamine administration: a review and evaluation of animal models of amphetamine psychosis. Brain Res. Rev. 396, 157-198.

Robinson T. E. and Camp D. M. (1991a) The feasibility of repeated microdialysis for within-subjects design experiments: studies on the mesostriatal dopamine system, in Microdialysis in the Neurosciences (Robinson T. E. and Justice J. B. Jr., eds), pp. 189234. Elsevier, Amsterdam.

Robinson T. E. and Camp D. M. (1991b) The effects of four days of continuous striatal microdialysis on indices of dopamine and serotonin neurotransmission in rats. $J$. Neurosci. Methods (in press).

Robinson T. E. and Whishaw I. Q. (1988) Normalization of extracellular dopamine in striatum following recovery from a partial unilateral 6-OHDA lesion of the substantia nigra: a microdialysis study in freely moving rat. Brain Res. 450, 209-224.

Robinson T. E., Jurson P. A., Bennett J. A., and Bentgen K. M. (1988) Persistent sensitization of dopamine neurotransmission in ventral striatum (nucleus accumbens) produced by past experience with $(+)$-amphetamine: a microdialysis study in freely moving rats. Brain Res, 462, 211-222.

Robinson T. E., Castañeda E., and Whishaw I. Q. (1990) Compensatory changes in striatal dopamine neurons following recovery from injury induced by 6-OHDA or methamphetamine: a review of evidence from microdialysis studies. Can. J. Psychol. 44, 253275.

Santiago M. and Westerink B. H. C. (1990) Characterization of the in vivo release of dopamine as recorded by different types of intracerebral microdialysis probes. Naunyn-Schmiedebergs Arch. Pharmacol. 342, 407-414.

Seiden L. S. and Ricaurte G. A. (1987) Neurotoxicity of methamphetamine and related drugs, in Psychopharmacology: The Third Generation of Progress (Meltzer H.Y., ed), pp. 359-366. Raven Press, New York.

Touchet N. and Bennett J. J. (1989) The metabolism of systemicallyadministered L-dihydroxyphenylalanine, by intact and dopamine-denervated striata, as revealed by brain microdialysis. Neuropharmacology 28, 1217-1222.

Ungerstedt U. (1984) Measurement of neurotransmitter release by intracranial dialysis, in Measurement of Neurotransmitter Release In Vivo (Marsden C. A., ed), pp. 81-105. John Wiley and Sons, New York.

Westerink B. H. and de V ries J. B. (1988) Characterization of in vivo dopamine release as determined by brain microdialysis after acute and subchronic implantations: methodological aspects. $J$. Neurochem. 51, 683-687.

Westerink B. H. and Tuinte M. H. (1986) Chronic use of intracerebral dialysis for the in vivo measurement of 3,4-dihydroxyphenylethylamine and its metabolite 3,4-dihydroxyphenylacetic acid. $J$. Neurochem. 46, 181-185.

Westerink B. H., Damsma G., Rollema H., De Vries J. B., and Horn A. S. (1987) Scope and limitations of in vivo brain dialysis: a 
comparison of its application to various neurotransmitter systems. Life Sci. 41, 1763-1776.

Winer B. J. (1971) Statistical Principles in Experimental Design. McGraw-Hill, New York.

Wolf M. E., Zigmond M. J., and Kapatos G. (1989) Tyrosine hydroxylase content of residual dopamine terminals following 6hydroxydopamine administration: a flow cytometric study. $J$. Neurochem. 53, 879-885.

Zetterström T., Sharp T., Collin A. K., and Ungerstedt U. (1988) In vivo measurement of extracellular dopamine and DOPAC in rat striatum after various dopamine-releasing drugs; implications for the origin of extracellular DOPAC. Eur. J. Pharmacol. 148, $327-334$.
Zhang W. Q., Tilson H. A., Nanry K. P., Hudson P. M., Hong J. S., and Stachowiak M. K. (1988) Increased dopamine release from striata of rats after unilateral nigrostriatal bundle damage. Brain Res. 461, 335-342.

Zigmond M. J., Acheson A. L., Stachowiak M. K., and Stricker E. M. (1984) Neurochemical compensation after nigrostriatal bundle injury in an animal model of preclinical parkinsonism. Arch. Neurol. 41, 856-861.

Zigmond M. J., Abercrombie E. D., Berger T. W., Grace A. A., and Stricker E. M. (1990) Compensations after lesions of central dopaminergic neurons: some clinical and basic implications. Trends Neurosci. 13, 290-296. 